\title{
Analyses of the Comprehension and Recognition of the Instructions in Educational Material for Disaster Prevention using an Assessment Model based on Cognitive and Motivational Factors
}

\author{
Erina Gyoba \\ Japan Society for the Promotion of \\ Science, International Research Institution \\ of Disaster Science, Tohoku University
}

\author{
Toshiaki Muramoto \\ International Research Institution of \\ Disaster Science, Tohoku University
}

\begin{abstract}
This paper focuses on how cognitive and motivational factors affected learners' comprehension of the instructions used in educational material for disaster prevention as well as their recognition. Based on a proposed double assessment model, we first classified the instructions in the learning material into the three types, knowledge-based $(K)$, rulebased(R), and skill-based (S) instructions, based on Rasmussen's model. Subsequently, students were asked to assess the learning material using the five motivational factors of Keller's ARCS-V model. Students' degrees of comprehension and recognition of the contents for each type of SRK were then assessed. We then investigated the extent of the motivational factors along with their correlation to the degree of comprehension and recognition for each cognitive type.

The results showed that the extent of the motivational factors largely differed according to the cognitive type of instructions, while these factors did not influence the degree of comprehension, especially in skill-based instructions. The motivational factors of 'volition', and 'confidence' had a strong and marginal effect respectively on the degree of recognition only in the case of rule-based instructions. These findings provide important suggestions for improving disaster educational programs in which increased motivational factors would adequately contribute to improved learning performances in versatile cognitive types.
\end{abstract}

\section{Introduction}

To create effective educational materials for disaster prevention, careful consideration should be given to the characteristics of human behaviour in emergency situations [1][2]. Although various kinds of education and training have been intensively implemented in Japan, such as hazard maps, evacuation manuals, emergency guidelines, and other disaster prevention plans prepared by both public and private organisations including educational institutions [3], research conducted following the 2011 Tohoku earthquake off the Pacific Coast in Japan reported that excessive adherence to the formulaic rule-based manuals sometimes disturbed people, preventing them from taking the appropriate actions to secure their safety [4][5]. On the other hand, most of the students in a district along the Pacific coast in the Tohoku area were able to escape the tsunami by spontaneously moving to higher and safer places, because they could translate the learned knowledge into adequate actions [6]. Thus, disaster prevention requires the development of the following three aspects in a well-balanced manner: 1) acquiring conceptual knowledge of disaster mechanisms and disaster histories, 2) understanding the rules to respond to emergencies, and 3) fostering the skills to take immediate and appropriate actions by applying the knowledge and rules in a flexible manner.

Furthermore, peoples' spontaneous motivation or awareness about the importance of disaster prevention is crucial in being prepared for substantial disasters [6]. Thus, the motivational effect is of great importance in preventive education for developing learners' abilities to use adequate knowledge for taking actions appropriately as required by the circumstances. As is generally known, learning motivation or the willingness to learn relates to learning outcomes [7]. However, the influence of motivational factors on learning performance on different cognitive types has not yet been well studied, especially in the field of education for disaster prevention. Developing an efficient program for such education is associated with the final goal of versatile safety education, notably 'the acquisition of ability to become aware of threatened situations and the application of such ability to avoid hazards' [3]. For this purpose, it is very important to investigate disaster education not only from the viewpoint of different cognitive characteristics, but also from that of the various motivational factors of people.

This study aims to provide insights into the designing principles of disaster prevention education in consideration of the differences in the cognitive characteristics of instructions along with different motivational factors. More specifically, this study investigated the influence of different motivational factors on learners' comprehension and recognition of different cognitive types of instructions included in educational materials for disaster prevention. The study employed a double assessment model to evaluate learning performance in relation to three different cognitive types of instructions and five 
different motivational factors. The present investigation comprised three different surveys of university students: a motivation survey, comprehension test, and recognition test as described below in greater detail.

\section{Assessment Model}

A twofold assessment model was proposed in order to evaluate how differences in motivation affected learners' comprehension and recognition of different cognitive types of instructions, as classified according to the hierarchical cognitive model. As illustrated in Figure 1, the double assessment model comprised two filtering approaches.

One of these approaches uses a three-way classification to categorise the instructions into three different cognitive types, namely knowledge-based $(\mathrm{K})$, rule-based $(\mathrm{R})$, and skill-based $(\mathrm{S})$ in accordance with the KRS hierarchical cognitive model [8] [9]. These three different cognitive types are hereafter called the KRS cognitive types. The filtering approach of this three-way classification enables us to evaluate the effectiveness of the instructions in each cognitive type when we investigate the instructional characteristics and learners' responses. We previously conducted a study on safety educational material, demonstrating the effectiveness of the three-way classification by KRS cognitive types in measuring the correspondence between instructional objectives and learners' cognitive responses [10].

The second filtering approach uses a five-way classification to investigate the five different motivational factors, namely attention (A), relevance $(\mathrm{R})$, confidence $(\mathrm{C})$, satisfaction $(\mathrm{S})$, and volition $(\mathrm{V})$ in accordance with the ARCSV motivation model [11][12]. These five motivational factors are commonly used in instructional design from the preparation and implementation stage to that of evaluation [13][14]. These five different motivation factors are hereafter called the ARCSV motivation factors.

The three-way classification of KRS cognitive types uses the following three criteria. In knowledgebased instructions, propositions and notions should be comprehended individually; in rule-based instructions, the procedural relationships between notions should be firmly established; in skill-based instructions, knowledge and rules should be transformed appropriately into automatic responses [8] [9] [10].

On the other hand, a classification method used to differentiate between the ARCSV motivational factors applies five criteria prescribed in the guidelines of the motivational design of instruction [11] [12] [13] [14]. Each of these five factors is defined as follows: attention refers to the interests aroused in learners when concepts or ideas are taught; relevance concerns how the lessons are relevant to the daily activities of the learner; confidence focuses on establishing positive expectations for achieving successful performances among learners; satisfaction refers to the pleasure or reward obtained from a learning experience; finally, volition relates to the will or desire that leads learners to implement the taught knowledge.

\section{Methods}

\subsection{Classification of the instructions in educational material by cognitive type}

For this study, an Internet-based learning program known as 'e-College Disaster Prevention and Risk Management' (URL: http://www.ecollege.fdma.go.jp/) created by the Japan Fire and Disaster Management Agency was classified into three (KRS) cognitive types of instructions. This classification was carried out with the participation of seven researchers majoring in cognitive psychology. At first, each researcher performed the task to evaluate each of the instructions according to the classification criteria shown in Table 1 by using a three-grade rating scale (from very low to very high) in order to assess the proximity of each instruction to each of the KRS cognitive types. Figure 2 illustrates a sample instruction from the material as well as the scale used for the classification task performed by each researcher.

Table 1. Classification criteria

\begin{tabular}{|c|c|}
\hline $\begin{array}{l}\text { Cognitive } \\
\text { type }\end{array}$ & $\begin{array}{c}\text { Classification } \\
\text { Criterion }\end{array}$ \\
\hline $\begin{array}{l}\text { Knowledge- } \\
\text { based } \\
\text { instruction }\end{array}$ & $\begin{array}{l}\text { If the paragraph contains descriptions } \\
\text { to promote a better understanding of } \\
\text { the causes and results, reasons, } \\
\text { definitions, or general concepts. }\end{array}$ \\
\hline $\begin{array}{l}\text { Rule-based } \\
\text { instruction }\end{array}$ & $\begin{array}{l}\text { If the paragraph contains explicit } \\
\text { descriptions of rules and procedures } \\
\text { that allow students to be aware } \\
\text { situational restrictions. }\end{array}$ \\
\hline $\begin{array}{l}\text { Skill-based } \\
\text { instruction }\end{array}$ & $\begin{array}{l}\text { If the paragraph contains descriptions } \\
\text { that promote students' actual skills in } \\
\text { task operations and emphasise the } \\
\text { achievement of automated behaviour } \\
\text { control. }\end{array}$ \\
\hline
\end{tabular}




\section{Classification filter based on Cognitive Hierarchical Model}

Classified

Cognitive reflections


Classification filter based on Motivational Factors

Figure 1. The proposed double assessment model using two filtering approaches to classify the hierarchical cognitive types and different motivational factors 
Example:

To prepare for a sudden earthquake, you should ensure escape routes are available. If there are many rooms in your house, you should place furniture in a room that family members rarely enter. If this is not possible, you should secure the furniture robustly or relocate it to make the space as secure as possible.

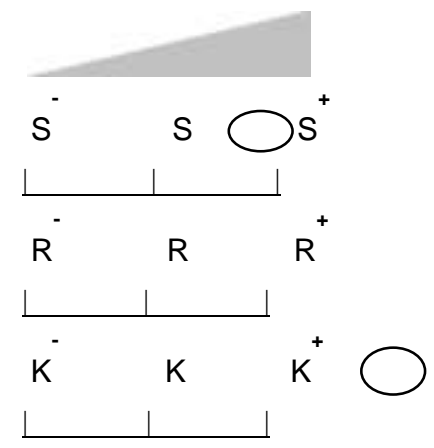

\section{Figure 2. Rating scale}

After the seven researchers completed the evaluation procedure, the rating scores were averaged according to the KRS cognitive types for each instruction. Thereafter, the cognitive type with the highest average score for a given instruction was determined to be the representative cognitive type for that particular instruction. In this way, each instruction was assigned to be one of the three cognitive types.

Following this classification procedure, a total of 51 instructions were categorised according to the three different cognitive types (17 instructions for each type). Table 2 provides examples of instructions for each of the three (KRS) cognitive types.

\section{Table 2. Examples of KRS instructions}

\section{Knowledge-based instruction}

When an earthquake occurs in shallow waters offshore, the bottom of the sea bulges or subsides. The surface of the sea then fluctuates with this diastrophism, and waves spread in every direction like a ripple made by a stone that has fallen in water, but on a massive scale. This is the mechanism of a tsunami.

\section{Rule-based instruction}

To prepare for a sudden earthquake, you should ensure escape routes are available. If there are many rooms in your house, you should place furniture in the room that family members rarely enter. If this is not possible, you should secure the furniture robustly or relocate it to make the space as secure as possible.

\section{Skill-based instruction}

If you feel shaking due to an earthquake when you are walking down the road, you must immediately keep away from tall fences and other objects at risk of falling down.
For the selected 51 instructions, the correlation coefficients among researchers' ratings were significantly high (ranging from 0.42 to 0.75 ), while each researcher individually rated the instructions in the present study. Therefore, it could be said that the KRS classification of the instructions was reliable.

\subsection{Participants and procedures of investigation}

The investigation was conducted with the participation of 137 students at Tohoku University between November 2011 and December 2012. The investigation included three different surveys as summarised as follows:

Table 3. Motivation Factors and Descriptions

\begin{tabular}{|l|l|}
\hline Factors & Description \\
\hline Attention & $\begin{array}{l}\text { It is attractive. } \\
\text { It is interesting. }\end{array}$ \\
\hline Relevance & $\begin{array}{l}\text { It is relevant to you. } \\
\text { It is meaningful for your future. }\end{array}$ \\
\hline Confidence & $\begin{array}{l}\text { It raises your confidence. } \\
\text { It increases your enthusiasm for } \\
\text { learning. }\end{array}$ \\
\hline Satisfaction & $\begin{array}{l}\text { It satisfies you. } \\
\text { You are content to learn it. }\end{array}$ \\
\hline Volition & $\begin{array}{l}\text { You can apply it soon. } \\
\text { It increases your will to implement } \\
\text { changes. }\end{array}$ \\
\hline
\end{tabular}

(i) Survey of motivation factors: In order to investigate the motivational characteristics of the 51 instructions according to the five-way classification, each of the 137 students was asked to rate each instruction using a three-grade evaluation $(-1,0,+1)$ for the following factors: attention, relevance, confidence, satisfaction, and volition based on the ARCSV model [11][12]. For this rating task, students were provided with a list of motivational factors along with descriptions (see Table 3 ) in order to estimate the intensity of the five different motivational factors. Consequence analysis was conducted in accordance with the ARCSV approach of the double assessment model.

(ii) Comprehension test: A test investigating the comprehension level of each instruction was given to 56 students who participated in the motivational survey. The test was carried out 3 weeks after the survey. For the true-or-false questions, 26 correct and 25 incorrect questions were drawn up and modified on the basis of the 51 original instructions. The order of the questions in the comprehension test was aligned differently from the order of instructions used in the survey of motivational factors. Table 4 shows example questions for each cognitive type.

(iii) Recognition test: Finally, we conducted a survey measuring the extent to which each instruction was recognized among another 75 students who participated in the motivational survey. The students were tested 3 weeks after the survey. Each of the students rated the strength of recognition of the original 51 instructions using a five-grade evaluation 
(very weakly recognized: 1 ; very clearly recognized:

5). The order of the questions in the recognition test was aligned differently from the order of instructions used in the survey of motivational factors.

\section{Table 4. Example questions in the comprehension test}

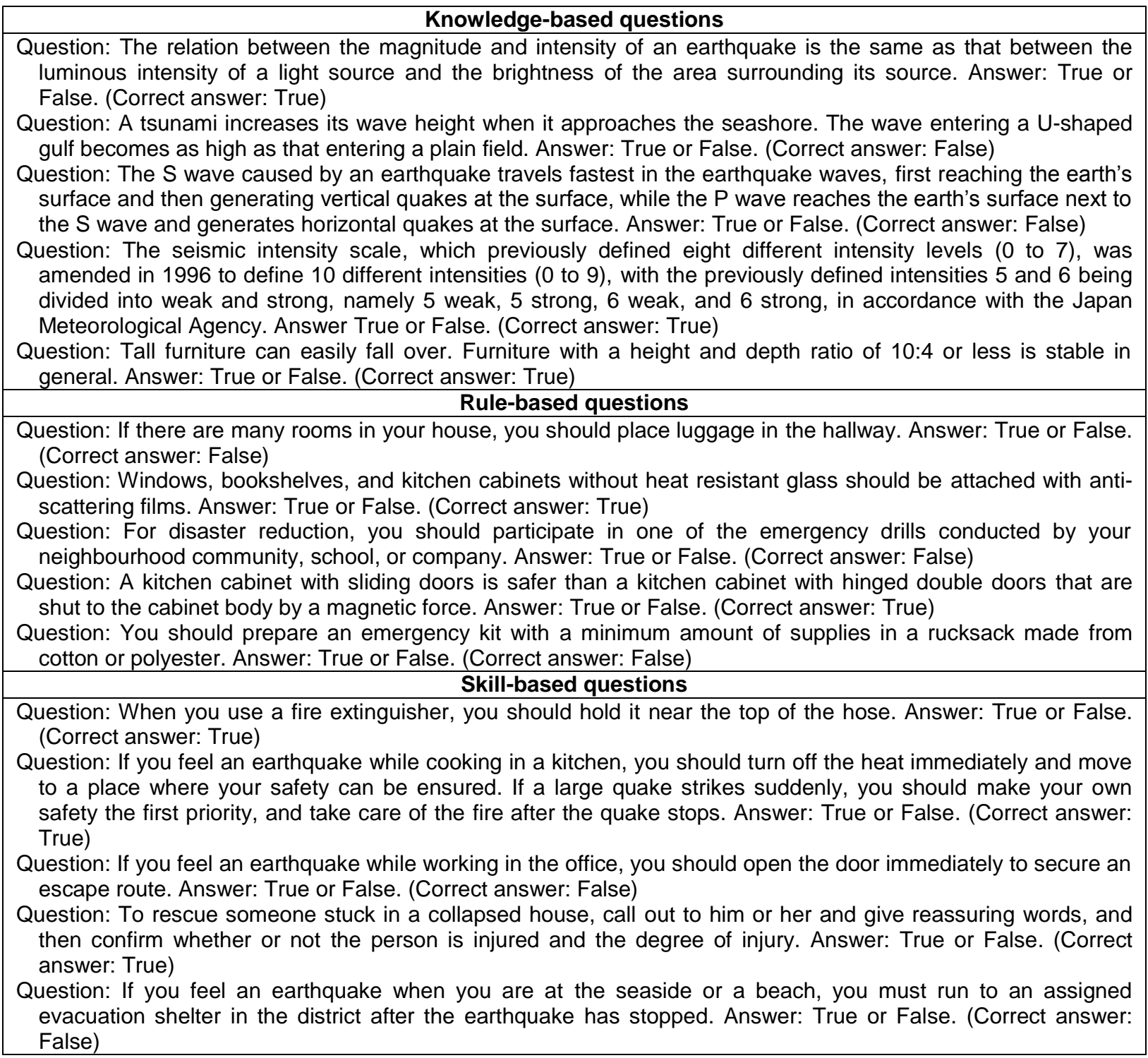

\section{Results}

\subsection{Magnitude of motivation for the instructions according to cognitive types}

Figure 3 shows the average rating scores for the five motivational factors (ARCSV) for the instructions according to cognitive type (KRS). Using a two-way ANOVA for the motivational factor and cognitive type, the main effects were both significant $(F(2,48)=6.030$, $p<.01$, and $F(4,192)=81.865, p<.01$, respectively $)$, while the interaction of the two factors was also significant $(F(8,192)=9.750, p<.01)$. Further analyses showed the significant simple main effect of each motivation factor in each cognitive type $(F(4,192)=37.018, p<$ $.01 ; F(4,192)=34.754, p<.01 ; F(4,192)=29.593, p$ $<.01$, respectively).
These results indicate that the intensity of the motivational factor largely differed depending on the type of cognitive characteristics. Based on the multiple comparisons, the following findings were obtained. In the instructions of the knowledge-based type, the intensity of the motivational factor 'satisfaction' was high as compared to the other motivational factors. In contrast, the rule-based instructions showed a high intensity for the motivational factors 'relevance', 'satisfaction', and 'confidence', while the factor 'attention' was low among the five motivational factors. The intensities of the motivational factors in the skill-based instructions were overall higher compared to the other cognitive types, especially for 'relevance', 'satisfaction', and 'confidence'. The motivational factor 'volition' was positive in both the rule- and skill-based instructions, while it was negative in the knowledge-based instructions. 


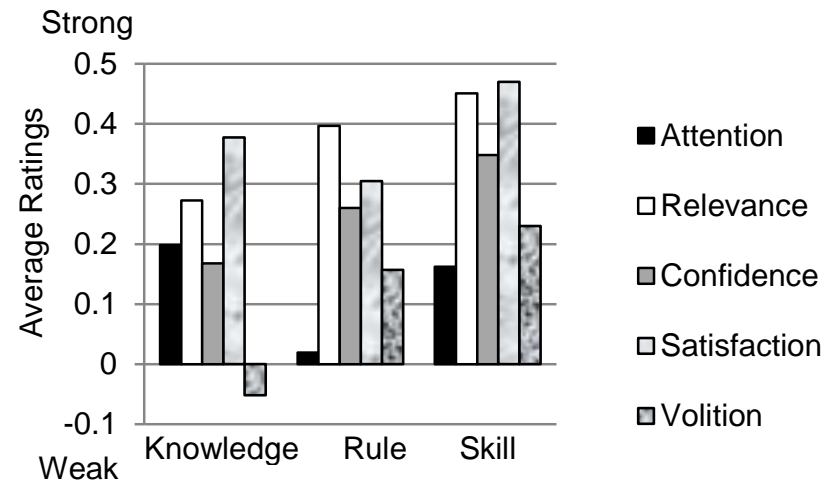

Figure 3. Ratings of the motivational factors in the instructions classified according to cognitive type

3.2. Comprehension and persistence of recognition of instructions according to cognitive types

Figures 4 shows the averages for the comprehension and recognition scores calculated by KRS cognitive type, respectively. The results of both the comprehension and recognition tests indicated that there was no significant difference in the average scores of the instructions as classified by KRS type for either survey.

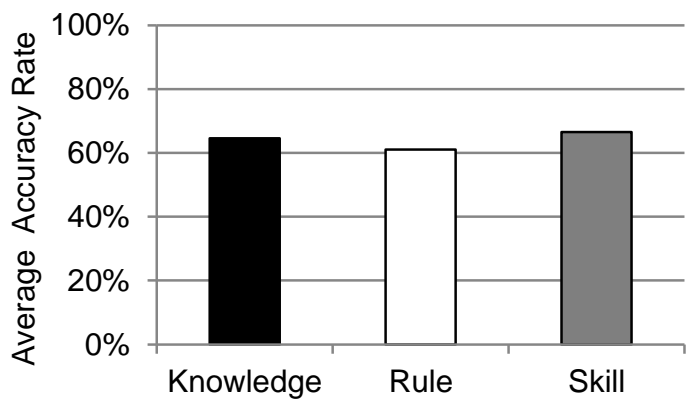

(a)

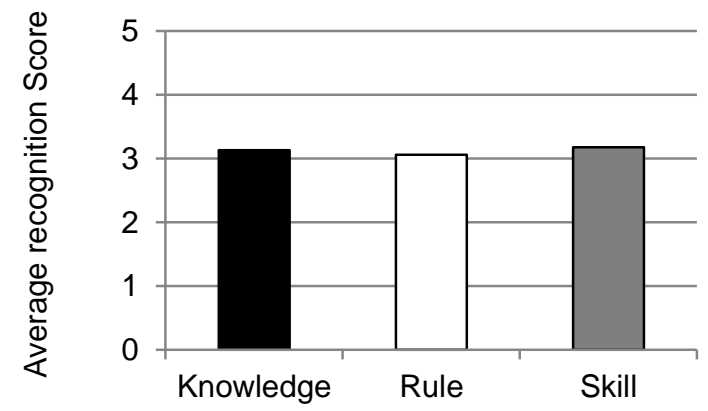

(b)

Figure 4. Average scores in the comprehension test (a) and in the recognition test (b) for each cognitive type
3.3. Correlation between comprehension scores and motivational factors in the instructions according to cognitive types

In order to examine the influence of the different motivational factors on the comprehension of instructions according to the different cognitive types, correlation coefficients were calculated based on the average comprehension scores for all of the students who took the comprehension test and the average motivation scores for those who participated in the survey on the five ARCSV motivational factors in the instructions. The analysis was conducted for each of the three cognitive types (KRS) (Figure 5).

The correlation between comprehension and the motivational factor 'confidence' was relatively higher in the knowledge-based instructions. However, the correlation of the comprehension score to the motivational factors in the rule-based instructions was rather low for all factors, although a slightly higher correlation was found in relation to 'attention'. In the skill-based instructions, the correlation between comprehension and motivation was negative for 'attention', 'relevance', and 'satisfaction', while it was very low for 'relevance'. However, none of these correlations between comprehension scores and motivational factor ratings reached significance for any of the KRS cognitive types.

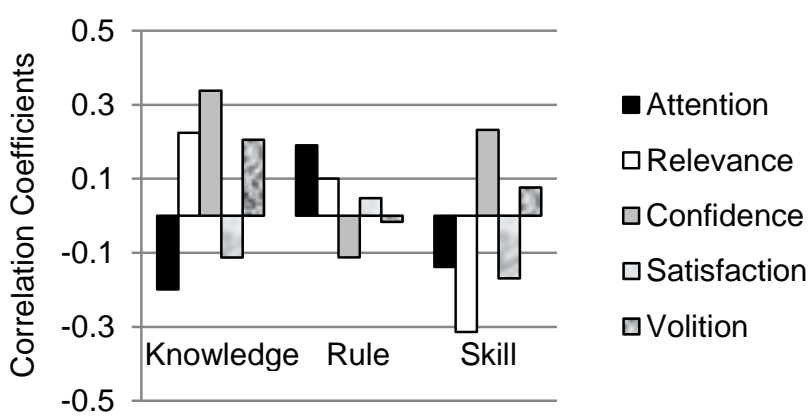

Figure 5. Correlation between comprehension scores and motivational factors for each cognitive type

\subsection{Correlation between recognition scores and motivational factors in the instructions according to cognitive types}

Figure 6 illustrates the correlation coefficients between the average recognition scores for all of the students who took the recognition test and the average motivational scores in the surveys on the five ARCSV motivational factors. The analysis was conducted for each of the three cognitive types (KRS). 


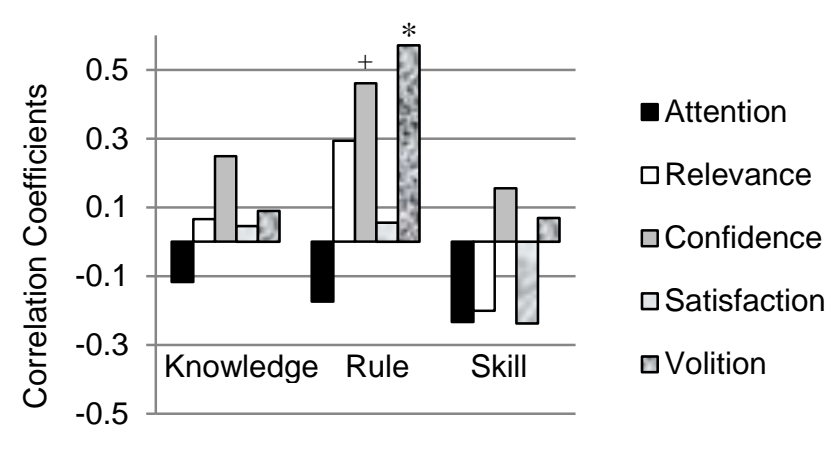

\section{Figure 6. Correlation between recognition scores and motivational factors for the cognitive types}

There was a significant correlation between the recognition scores and the motivational factor 'volition' $(r=0.57, d f=17, p<.05)$ and only a marginally significant correlation between the recognition scores and 'confidence' $(r=0.46, d f=17, p<.10)$ in the rulebased instructions. Interestingly, in terms of the comprehension test, the correlations for these two motivational factors were negative, while for the recognition test, both were significantly or marginally significantly positive. In the knowledge-based instructions, the correlation of the recognition test with the motivational factor 'confidence' was relatively higher, while it was negative for 'attention'. In the skill-based instructions, the correlations between the recognition test and motivation factors were not high, being negative for 'attention', 'relevance', and 'satisfaction'.

\section{Discussion}

Our results reveal that the factors driving learners' motivation were different depending on the cognitive type of the instructions, as shown in Figure 3. While the motivational factors 'satisfaction', 'confidence', and 'relevance' had relatively higher scores in all cognitive instruction types, rather low scores were found for the motivational factor 'volition' in the knowledge-based instructions and 'attention' in the rule-based instructions. On the other hand, all five motivational factors had high scores in the skill-based instructions as compared with the knowledge- and rule-based instructions. This suggests that the skillbased instructions could be more likely to motivate learners in comparison with the other cognitive types of instructions.

On the other hand, there was no difference among the three different cognitive types for the results of the comprehension test or recognition test, with both tests being conducted 3 weeks after the motivation survey (see Figure 4). Moreover, these results showed a similar tendency, notably that learners' comprehension of the instructions for each cognitive type could correspond to the recognition of these instructions when motivational factors were not considered.

A close examination of the relationship between the motivational factors and the recognition of the instructions by cognitive type demonstrated that the factor 'volition' showed a significantly high correlation and confidence a marginally significant correlation in the rule-based instructions (see Figure 6). This suggests that rule-based instructions are more likely to motivate learners with respect to volition, such as the notion of the 'willingness to do'. Furthermore, rule-based instructions, which tended to encourage confidence that led to successful performances in the tests, also enhanced the recognition of the instructions.

From the analysis of the correlation between the motivational factors and the comprehension and recognition scores by cognitive type, it was found that the correlation patterns were similar in the knowledgeand skill-based instructions (see Figure 5 and 6). It can therefore be said that, in both types of instructions, the correlation pattern for the comprehension scores was more amplified than that of the recognition scores. More specifically, the motivation factor 'confidence' had a relatively higher correlation with the recognition scores and a much higher correlation with the comprehension scores in the knowledge- and skillbased instructions. Thus, it can be argued that the instructions stimulating learners' confidence should be promoted in learning materials with regard to both knowledge- and skill-based instructions.

On the contrary, the rule-based instructions showed no amplified correlations between the motivation factors and the comprehension scores. In fact, the correlations between the motivation factors "Attention", "Confidence" and "Volition" and the comprehension scores showed opposite tendencies to those for the recognition scores. Notably, the motivation factor "volition" and "confidence" showed no more significant correlation with the comprehension in the rule-based instruction. Rather, these two motivational factors had negative correlations. From this viewpoint, it should be considered that the contribution of motivation factors to the recognition did not have a straightforward effect on the enhancement of comprehension in the rulebased instructions.

In the skill-based instructions, both the comprehension and recognition scores had very weak or sometimes negative correlations with all of the motivational factors, with the exception of 'confidence' and 'volition'. This could be explained by the following two reasons. Firstly, the motivation scores for the skill-based instructions were all higher compared with the other two cognitive types of instructions, as shown in the results of the motivation survey (see Figure 3). However, the average scores of the recognition and comprehension tests for the skillbased instructions were almost the same as those 
obtained for the other two cognitive types. This could result in lower correlations between the motivational factors and the recognition and comprehension scores for the skill-based instructions in comparison with the knowledge- and skill-based ones. Secondly, the investigation procedure of the present study did not involve practical disaster prevention trainings. This could have hampered learners' motivation to develop the learning outcomes for the skill-based instructions.

\section{Conclusion}

This study aimed to investigate the characteristics of education materials used for disaster prevention based on the proposed double model of human hierarchical cognitive theory and learning motivational theory. By using this method to classify instructions in accordance with the KRS cognitive types in conjunction with a method to classify motivational factors in terms of ARCSV, empirical evidence was obtained in order to confirm the interactive influences of the cognitive and motivational factors on learning disaster prevention instructions.

As a result, motivation factors were found to be different depending on the cognitive type of the instructions (KRS). The contribution of five motivational factors to the recognition of the instructions and their increased comprehension was not the same in the three cognitive types. Notably, the motivation factor 'volition' had a significant correlation and confidence a marginally significant correlation with the recognition of rule-based instructions. The results of this investigation suggest that:

(i) The instructions that stimulate learners' confidence promote learning of both knowledge- and skill-based instructions.

(ii) Rule-based instructions stimulate the motivation of learners more with respect to volition, such as the notion of the 'willingness to have to do', and confidence related to successful achievement

(iii) Highly motivated learning does not always contribute to enhancing the comprehension of skill-based instructions. The investigation procedure of this study did not involve practical disaster prevention trainings, which may have hampered learners' motivation to work on the actual learning outcomes for the skill-based instructions.

Future studies should aim to determine educational programs in which increased motivational factors adequately contribute to learning performance in versatile cognitive types. For example, it should be examined how the visual (pictorial) presentation of instructions or repetitive practices and training would help the motivational factors to contribute to creating positive correlations with the comprehension and recognition of instructions for all three cognitive types.

\section{Acknowledgements}

This research was supported by a postdoctoral fellowship from the Japan Society for the Promotion of Science for Young Scientists (244610), which was awarded to the first author of this research study, Erina Gyoba. The authors would like to thank Prof. F. Imamura, Prof. T. Yamamura, Associate Prof. Y. Ninose, and Dr. H. Shibata for their kind support.

\section{References}

[1] E, Gyoba (2012), 'Characteristics of the Behaviors to Collect Information from Available Media in the Students of Universities Located in the Damaged and Non-Damaged Prefectures Around the Period of the 2011 off Pacific Coast of Tohoku Earthquake', Journal of Disaster Research, 7(6), Fuji Technology Press, Tokyo, pp.786-792

[2]F. Imamura (2001) 'Extensive Damage Caused by Tsunami: Prediction and Countermeasure'(in Japanese), Journal of Geography,110(6), Tokyo Geographical Society, Tokyo, pp.828-837

[3] Board of Education in Miyagi Prefecture (2009) 'Foundation of Education for Disaster Prevention in Miyagi Prefecture' (in Japanese), Information about Disaster and Safety;

http://www.pref.miyagi.jp/uploaded/attachment/15509.pdf (15,December 2012)

[4] T. Izumi and H. Yamaguchi (2011) 'About Education for Disaster Prevention Training in the New-comer Training'(in Japanese), Center of Education in Wakayama Prefecture; URL:http://www.wakayama-edc.big-

u.jp/kenkyukiyo23/H23/H23-6.pdf (3, September 2012).

[5] T. Katada (2012) 'The Attitudinal Education for Disaster Prevention which Protected Children: Learning from Proactive Behaviors of Pupils and Students Who Survived the Severe Tsunami Disaster in Kamaishi' (in Japanese), Journal of Japan Society for Disaster Information Studies, Japan Society for Disaster Information Studies, Tokyo, pp. $37-42$

[6] F. Imamura (2011)'Risk of Natural Disaster and Education for Disaster Prevention: Living in Geo-sphere'(in Japanese), in F. Imamura (Eds), Development of Education for Disaster Prevention, Toshindo: Tokyo, pp.3-17

[7] R.M. Gagné, W.W. Wagner, K.C Golas and J.M. Keller, (2005) Principle of Instructional Design $\left(5^{\text {th }} e d\right)$, CA: Wadsworth/ Thomson Learning, Belmont

[8] J. Rasmussen (1986) Information Processing and Human-Machine Interaction- An Approach To Cognitive Engineering, Elsevier Science Publishing Company, Inc., New York

[9] E. Gyoba and S. Iwasaki (2007) 'Illustrative Explanation of the Cognitive Behavior levels and Error types' (in Japanese), Educational Informatics, 5, Graduate School of Educational Informatics Tohoku University, Sendai, pp.71-79 
[10] E. Gyoba and S. Iwasaki (2007)'Analyses of Safety Learning Material for Preliminary Experimental Class of Natural Science in University Education based on Human Hierarchical Performance Model' (in Japanese), Journal of Educational Technology, 31, Japan Society for Educational Technology, Tokyo, 2007, Tokyo, pp.29-32

[11] J.M. Keller (2008) First principle of motivation to learn and e-learning, Distance Education, 29(2), Open and Distance Learning Association of Australia, Sydney, pp.175176

[12] J.M. Keller (1987) 'Development and use of the ARCS model of motivational design', Journal of Instructional Development, 10(3), Association for Educational Communications and Technology, Washington, pp.2-10.

[13] C. Kogo and K. Suzuki (1998)'Making an Evaluation Sheet for Courses and Learning Materials Based on ARCS Motivation', (in Japanese) Proceedings of Annual Conference of Japan Society for Educational Technology; http://www2.gsis.kumamoto-u.ac.jp/ idportal/wpcontent/uploads/jset98.pdf (19, December. 2012)

[14] K. Suzuki (2009) 'Research Trends to Support the Design of "Willing to Learn" '(in Japanese), Proceedings of $16^{\text {th }}$ Annual Meetings of Japan Association for Education Media Society; http://www2.gsis.kumamotou.ac.jp/ idportal/wpcontent/uploads/a910jaems_nigata.pdf (20, December, 2012) 